\title{
ON ANALYTICITY OF LOCAL RESOLVENTS AND EXISTENCE OF SPECTRAL SUBSPACES
}

\author{
CHE-KOA FONG
}

\begin{abstract}
We present some sufficient conditions for a function from an open set in $\mathbf{C}$ into a Hilbert space $H$ such that $(T-z) f(z)=x(T \in B(H)$ and $x \in H$ ) to be analytic. As an application we show that hyperinvariant subspaces exist for certain class of operators.
\end{abstract}

Let $T$ be a bounded operator on a Hilbert space $H$. Suppose $f$ is a vectorvalued mapping from an open set $U$ in the complex plane $\mathrm{C}$ into $H, y$ is a vector in $H$, and $(T-z) f(z)=y$ for all $z$ in $U$. We ask what additional conditions force $f$ to be analytic. For example, a recent work of Stampfli and Wadhwa [6] showed that if $T$ is dominant and $f$ is bounded, then $f$ is analytic. (Also see [5].) In this note, we present some circumstances under which $f$ is analytic. As an application we give a sufficient condition for the existence of hyperinvariant subspaces.

For a Hilbert space $H$, we shall write $B(H)$ for the set of all bounded operators on $H$. Let $T \in B(H)$ and $F$ be a compact set in C. We shall write $X_{T}(F)$ for the linear manifold consisting of those $x$ in $H$ such that $(T-z) f(z)$ $\equiv x$ for some analytic vector-valued function $f$ from $\mathbf{C} \backslash F$ into $H$. For convenience, we call the closure of $X_{T}(F)$ a spectral subspace of $T$. Obviously, a spectral subspace of $T$ is always hyperinvariant for $T$; that is, it is invariant for every operator commuting with $T$. For basic properties of spectral manifolds $X_{T}(F)$ we refer to [1]. We shall write $\operatorname{Sp}(T)$ for the spectrum of $T$ and $\Pi(T)$ for the approximate point spectrum of $T$. For the definition and basic properties of approximate point spectra, see Chapter 8 in [2]. For $F \subseteq \mathbf{C}$, we write $F^{*}$ for $\{\bar{z}: z \in F\}$.

Proposition 1. If $T \in B(H)$ and $y \in \bigcap_{z \in U}(T-z) H$ where $U$ is an open set in $\mathbf{C}$ such that $U \cap \Pi(T)=\varnothing$, then $z \rightarrow(T-z)^{-1} y$ is an analytic vectorvalued function.

Proof. For convenience, write $f(z)=(T-z)^{-1} y(z \in U)$. (This function is uniquely defined, by the hypothesis on $y$.) First we show that $f$ is bounded on compacta. If not, there exists a convergent sequence $\left\{z_{n}\right\}$ in $U$ such that $z_{0}=\lim _{n} z_{n} \in U$ and $\lim _{n}\left\|f\left(z_{n}\right)\right\|=\infty$. Let $x_{n}=\left\|f\left(z_{n}\right)\right\|^{-1} f\left(z_{n}\right)$. Then

Received by the editors September 15, 1976 and, in revised form, October 8, 1976.

AMS (MOS) subject classifications (1970). Primary 47A10, 47A15; Secondary 47B20.

Key words and phrases. Approximate point spectrum, spectral subspace, $M$-hyponormal operator.

๑ American Mathematical Society 1977 


$$
\left\|x_{n}\right\|=1 \text { and }
$$

$$
\begin{aligned}
\left\|\left(T-z_{0}\right) x_{n}\right\| & \leqslant\left\|\left(T-z_{n}\right) x_{n}\right\|+\left\|\left(z_{n}-z_{0}\right) x_{n}\right\| \\
& =\left\|f\left(z_{n}\right)\right\|^{-1}\|y\|+\left|z_{n}-z_{0}\right| \rightarrow 0
\end{aligned}
$$

as $n \rightarrow \infty$. This contradicts the fact that $z_{0} \notin \Pi(T)$.

Let $z_{0} \in U$. Then, for $z \neq z_{0}$, we have

$$
\left(T-z_{0}\right) g(z)=f(z),
$$

where

$$
g(z)=\left(z-z_{0}\right)^{-1}\left(f(z)-f\left(z_{0}\right)\right) .
$$

Since $z_{0} \notin \Pi(T)$, the operator $T-z_{0}$ is bounded below and thus has a bounded inverse when it is considered as a linear map from $H$ onto its range $\left(T-z_{0}\right) H$ (which is closed). (We shall designate this inverse by $\left(T-z_{0}\right)^{-1}$.) From (*) we see that $g$ is bounded on a neighborhood of $z_{0}$ and hence, by (**), $f$ is continuous at $z_{0}$. We have shown that $f$ is a continuous function. By (*) again, we have

$$
\lim _{z \rightarrow z_{0}} g(z)=\lim _{z \rightarrow z_{0}}\left(T-z_{0}\right)^{-1} f(z)=\left(T-z_{0}\right)^{-1} f\left(z_{0}\right) .
$$

Hence, by (**), $f$ is differentiable at $z_{0}$. The proof is complete.

REMARK. Since the boundary of $\operatorname{Sp}(T)$ is contained in $\Pi(T)$ and is a closed set, the open set $U$ in the above proposition is a disjoint union of two open subsets $U_{1}$ and $U_{2}$ with $U_{1} \subset \operatorname{Sp}(T)$ and $U_{2} \cap \operatorname{Sp}(T)=\varnothing$. It is well known that the map $z \mapsto(T-z)^{-1}$ is analytic on $U_{2}$. Hence our interest of the proposition is the case when $U \subset \operatorname{Sp}(T) \backslash \Pi(T)$.

Corollary. If $T \in B(H), F$ is a compact set in $\mathrm{C}$ and $R \supseteq \Pi(T)$, then $X_{T}(F)$ is closed.

Proof. In fact, by Proposition 1, we have

$$
X_{T}(F)=\bigcap_{z \in \mathbf{C} \backslash F}(T-z) H
$$

where each $(T-z) H$ is closed.

Proposition 2. Let $T \in B(H), F$ be a compact set in $\mathrm{C}$ and $x \in H$. If $f: \mathbf{C} \backslash F \rightarrow H$ is a bounded vector-valued function such that $(T-z) f(z) \equiv x$ and $X_{T^{*}}\left(F^{*}\right)$ is dense in $H$, then $f$ is analytic.

Proof. Since $f$ is bounded, it suffices to show that the map $z \mapsto(f(z), y)$ is analytic for each $y$ in a dense subset of $H$. Let $y \in X_{T^{*}}\left(F^{*}\right)$. Then there is an analytic function $g: \mathbf{C} \backslash F^{*} \rightarrow H$ such that $\left(T^{*}-\bar{z}\right) g(\bar{z})=y$ for $z \notin F$. Hence, for $z \notin F$, we have 


$$
(f(z), y)=\left(f(z),\left(T^{*}-\bar{z}\right) g(\bar{z})\right)=((T-z) f(z), g(\bar{z}))=(x, g(\bar{z})) .
$$

Clearly $z \mapsto(x, g(\bar{z}))$ is analytic. The proof is complete.

REMARK. The above results can be easily generalized to operators on Banach spaces.

As an application of Proposition 2, we have the following:

Proposition 3. Let $T \in B(H)$. Suppose: (1) there is a nonzero invariant subspace $K$ of $T$ such that $T \mid K$ is a normal operator, and (2) there is a nonzero positive operator $P$ such that $(T-z)^{*}(T-z) \geqslant P^{2}$ for all $z \in \mathbf{C}$. Then $T$ has a nontrivial spectral subspace.

Proof. Let $y \in H$ be a vector such that $x=P y \neq 0$. By Putnam [3, Theorem 6], there exists a bounded vector-valued function $f: \mathbf{C} \rightarrow H$ such that $\left(T^{*}-z\right) f(z)=x$.

Let $E$ be the resolution of identity for $T \mid K$ and $\mathscr{D}$ be the collection of all closed discs $D$ in $\mathbf{C}$ such that $\operatorname{Sp}(T \mid K) \cap$ (interior of $D) \neq \varnothing$. For $D \in \mathscr{D}$, we have $X_{T}(D) \supseteq E(D) K \neq\{0\}$. Hence it suffices to show that $X_{T}(D)$ is not dense in $H$ for some $D$ in $\mathscr{D}$. Suppose otherwise. Then, by Proposition 2, for each $D \in \mathscr{D}, f$ is analytic on $\mathbf{C} \backslash D^{*}$. Hence $f$ is a bounded entire function with

$$
\lim _{|z| \rightarrow \infty} f(z)=\lim _{|z| \rightarrow \infty}\left(T^{*}-z\right)^{-1} x=0 .
$$

By Liouville's theorem, $f=0$, contradicting $x \neq 0$ and $\left(T^{*}-z\right) f(z)=x$. The proof is complete.

Corollary. Let $T_{1} \in B\left(H_{1}\right)$ and $T_{2} \in B\left(H_{2}\right)$. Suppose: (1) there is a nontrivial invariant subspace $K$ of $T_{1}$ such that $T_{1} \mid K$ is a normal operator, and (2) $T_{2}$ is a nonscalar M-hyponormal operator (that is,

$$
\left(T_{2}-z\right)\left(T_{2}-z\right)^{*} \leqslant M\left(T_{2}-z\right)^{*}\left(T_{2}-z\right)
$$

for all $z$ in $\mathbf{C})$. Then $T=T_{1} \oplus T_{2}$ has a nontrivial spectral subspace.

Proof. Let $P$ be the positive square root of $\left(T_{2}^{*} T_{2}-T_{2} T_{2}^{*}\right)^{2}$. If $P=0$, then $T_{2}$ is normal and we are done. Hence we may assume that $P \neq 0$. By Radjabalipour [5, Theorem 2], there is a positive number $k$ such that $(T-z)^{*}(T-z) \geqslant k P^{2}$ for all $z$ in C. Now the corollary follows from Proposition 3.

REMARK. We do not know in the above corollary if $T_{1}$ and $T_{2}$ separately have nontrivial hyperinvariant subspaces (provided they are nonscalar operators).

ACKNowledgement. The author would like to thank M. D. Choi for his help which leads to a simplified proof of Proposition 1.

\section{REFERENCES}

1. I. Colojoară and C. Foiaş, Theory of generalized spectral operators, Gordon and Breach, New York, 1968. 
2. P. R. Halmos, A Hilbert space problem book, Van Nostrand, Princeton, N.J., 1967. MR 34 \#8178.

3. C. R. Putnam, Hyponormal contractions and strong power convergence, Pacific J. Math. 57 (1975), no. 2, 531-538. MR 52 \# 1393.

4. M. Radjabalipour, Ranges of hyponormal operators (to appear).

5. $\longrightarrow$, On majorization and normality of operators, Proc. Amer. Math. Soc. 62 (1977), $105-110$.

6. J. G. Stampfli and B. L. Wadhwa, On dominant operators (to appear).

Department of Mathematics, University of Toronto, Toronto, Ontario msS 1Ai, CANADA 